\title{
Speed Control and Frequency Conversion Interference Treatment of Variable Frequency Ventilator
}

\author{
Sun Guoli \\ Jiangsu Province Xuzhou Technician Institute, Xuzhou, Jiangsu, 221000
}

Keywords: Fan, Frequency Conversion, Interference

\begin{abstract}
Frequency converter is a device that regulates the speed of power equipment such as motor by adjusting the power supply frequency. It is the best way of speed regulation at present. It is applied more and more widely in large industrial and mining enterprises. But by the frequency converter to the electronic circuit of frequency harmonic interference generated in the process a lot of interferences on the grid and microelectronic devices, corresponding measures are not taken under the condition of microelectronic devices in a state of paralysis, therefore, to solve the harmonic interference is an important topic. By analyzing the working principle of frequency converter and the cause of harmonic interference, this paper puts forward relevant measures to solve harmonic interference in on-line monitoring system of fan performance.
\end{abstract}

\section{The principle of variable frequency speed regulation and its inverting mode}

\subsection{The principle of variable frequency speed regulation}

According to the literature, AC asynchronous motor is a motor driven by the electromagnetic induction generated by the current passing through the magnetic field line, and the motor speed is directly proportional to the AC frequency.

$$
n=\frac{60 f_{1}}{p}(1-s)=\frac{60 \omega_{1}}{2 \pi p}(1-s)
$$

In the formula:

$f_{1}$-Alternating current frequency loaded into the stator winding of an asynchronous motor

$\omega$-Angular frequency of alternating current;

$p$-The logarithm of the magnetic pole of an asynchronous motor;

$n_{s}=\left(n_{s}-n\right) / n_{s}=\left(\omega_{1}-\omega\right) / \omega_{1}-$ Change rate of motor running;

$n_{s}=60 f_{1} / p=60 \omega_{1} / 2 \pi p$ - Synchronous speed of asynchronous motor.

From formula (1) it follows that the frequency $f_{1}$ of the alternating current applied to the stator windings of the motor is varied by smoothing. You can play to regulate asynchronous motor rotor speed $n$ and synchronous speed $n_{s}$ purpose, thereby changing the motor speed, which is the basic principle of the inverter work.

\subsection{Inverting mode of frequency converter}

The constant amplitude PWM type indirect frequency conversion technology is a more common frequency modulation technology, which consists of diode inverter, rectifier circuit and filter capacitor. After three phase alternating current is rectified through diode, the DC voltage is obtained, and then fed to the inverter, and then the voltage regulation is completed by adjusting the pulse width and switching frequency of the inverter, and the three-phase voltage and the frequency modulated alternating current are output, which are used for the load [1-2].

Taking the single-phase inverter circuit as an example, the realization of inverter is introduced. The way of PWM control is to change the frequency of two sets of power transistors (T1, T4, T3, T6, another group) alternately, so as to change the frequency of the output pulse waveform of the inverter. 
Another mode of modulation is the SPWM type, which is added to the control end of the switch element with a triangular carrier and a sinusoidal modulation wave. By comparing the relationship between the two kinds of wave and time, we decide the on-off of switch devices and achieve the purpose of modulating the pulse width. Here is a detailed introduction in document [3].

\section{Energy saving analysis of fan frequency conversion operation}

There is a linear relationship between the fan speed $\mathrm{n}$ and the flow rate, the pressure $\mathrm{H}$ and the axis power $\mathrm{N}$ respectively, the two square relations and the three square relations.

$$
\begin{aligned}
& q_{1}=q_{2}\left(n_{1} / n_{2}\right) \\
& H_{1}=H_{2}\left(n_{1} / n_{2}\right)^{2} \\
& N_{1}=N_{2}\left(n_{1} / n_{2}\right)^{3}
\end{aligned}
$$

When the speed of fan is constant and the opening of the air door is reduced, that is, increasing the resistance of the pipe network. The resistance characteristic of the pipe network changes to 3 . The working point of the ventilation system moves from E to F. At this time, the wind pressure increases, and the shaft power $\mathrm{N} 2$ is directly proportional to the area FH2OQ2. If the flow regulation based on frequency converter, fan speed reduced from $n_{1}$ to $n_{2}$, the H-Q curve of $n_{2}$ speed is 4; the operating point from $\mathrm{E}$ to $\mathrm{D}$, in the same volume of $\mathrm{Q} 2$, pressure $\mathrm{H} 3$ greatly reduce the area of DH3OQ2 is obviously reduced; power loss of $\Delta N$ product with Q2 and save area $\Delta H$ is proportional to the. It can be seen that the variable frequency speed regulation can save energy.

\section{Harmonic interference and harm of frequency converter}

\subsection{Generation of harmonic interference in frequency converter}

The main circuit of frequency converter is generally the AC direct to AC structure. The external $380 \mathrm{~V} / 50 \mathrm{~Hz}$ input power frequency power is controlled by three phase bridge path and can be rectified into DC voltage signal. After filtering capacitor filter and high-power transistor switching element, the inverter is converted to variable frequency AC signal. In rectifier circuit, the waveform of input current is irregular rectangular wave. The waveform is decomposed into fundamental and all harmonics according to Fu Liye series, and the high-order harmonics will interfere with the input power supply system. In the inverter output circuit, the output current signal is modulated by the PWM carrier signal [4]. For GTR high power inverter, the carrier frequency of PWM is $2 \sim 3 \mathrm{kHz}$, while the highest carrier frequency of IGBT high power inverter is $15 \mathrm{kHz}$. In the same way, the current signal of the output circuit can be decomposed into the fundamental and other harmonics with only the sine wave, and the high harmonic current is directly interfered with the load. In addition, the high order harmonic current is also radiated into the space through the cable to interfere with the adjacent electrical equipment.

\subsection{Common methods to suppress harmonic interference}

The transmission way of harmonics is conduction and radiation. To solve the conducted interference is to filter or isolate the high-frequency current transmitted in the circuit, and to solve the radiation interference is to shield the radiation source or the jammed line. The commonly used method [5]:

1) The power supply of the frequency conversion system is independent of the power supply of other devices, or the isolation transformer is installed on the input side of the frequency converter and other electrical equipment, and the harmonic current is cut off.

2) A suitable reactor or a harmonic filter should be connected to the input side and the output side of the inverter. The composition of the filter must be LC type, which can absorb harmonic and increase the impedance of the power or load, so as to achieve harmonic suppression. 
3) Between the motor and inverter cable wear steel pipe laying or armoured cable, and other weak signals in different cable trench avoiding radiation interference.

4) A shield line is used in the signal line, and a certain distance (at least $20 \mathrm{~cm}$ ) is separated from the control line of the main circuit of the inverter, and the radiation interference is cut off.

5) The frequency converter uses a special grounding wire and is grounded with a short line. The ground line adjacent to other electrical equipment must be separated from the inverter and use a short line. This can effectively suppress the radiation interference of current harmonics to adjacent equipment.

\section{Interference and solution of harmonic interference to monitoring system under variable frequency environment}

\subsection{Sources and prevention measures of electromagnetic interference in industrial environment}

The main source of electromagnetic interference

1) Radiation interference from space

Space radiation field is generated by electrical equipment of power network, the transient process, lightning, television, radar, high frequency induction heating equipment, usually called by local radiation interference, shielding and high pressure shielding cable and related equipment discharge protection elements [6].

2) Interference from the outside of the system

The interference from the external lead of the system is usually referred to as the conduction interference [7]. The way of interference transmission is through power supply and signal line. This type of interference is more common and serious in the industrial field of our country. There are three main types:

(a) Power interference. A lot of equipment monitoring system of normal power supply from the power supply, the power grid covering a wide range, it will have all the space electromagnetic interference on the line induced voltage and current, especially large power equipment to stop the AC-DC transmission device caused by harmonic and transient impact, are transmitted through the transmission lines of primary power supply. For power interference, it is usually solved by the measures of isolating power supply for the power supply of important equipment.

(b) The interference introduced by the signal line. The monitoring system has a large number of signal transmission lines connecting all the sensors and control devices, and the signal line plays the role of transmitting effective signals. However, there are always some irrelevant external disturbance signals accompanied by monitoring cables. The interference way has two kinds: one is through the power grid disturbance power supply or a common instrument in series, which is often ignored; two is the signal line by space electromagnetic radiation induced interference in the routing process, namely the external inductive interference signal line, this is very serious, and caused a reduction of I/O signal abnormalities, accuracy the more serious damage of components.

(c) Grounding system interference. The correct grounding mode can effectively suppress electromagnetic interference. The wrong grounding will introduce external interference signals into the system, which will affect the normal operation of the monitoring system. The system ground lines include the system, the shielding, the communication and the protection. The disturbance of the grounding system to the monitoring system is mainly due to the uneven distribution of potential in each grounding point, and the potential difference between the different grounding points, causing the earth loop current, which affects the normal operation of the system [8-9].

Common preventive measures for electromagnetic interference in industry:

Electromagnetic interference seriously affects the normal operation of the industrial system, and the situation will seriously damage the industrial production. Therefore, in order to ensure the system avoid or reduce the interference in the electromagnetic environment, must take relevant measures in the design stage, the main control measures in three aspects: first, to suppress the interference source, second transmission cut off or attenuation of electromagnetic interference, the 
third is to improve the device and system's anti-interference ability, common concrete measures the following points.

1) Consider the interference from the overall design of the monitoring system and lead to prevent radiation shielding, electromagnetic interference; external wiring isolation, filtering processing, especially the power cable, control cable, cable monitoring should be layered arrangement, to prevent the outer lead by introducing EMI, for the design and installation of grounding and the grounding device correctly, improve the grounding system; when necessary by means of software through software filtering technology, improve the anti-interference ability of the system, increase safety and reliability.

2) To design and select a good power supply to suppress the interference introduced by the power grid. The power of the computer, the power of the I/O module, the power of a part of the sensor and the power of the transmitter are the equipment that the external interference signal is easy to enter. At present, the commonly used measure is to use a UPS stable voltage source with good isolation performance. However, due to the wide distribution of sensors and transmitters and the complete isolation of space, it is necessary to choose various instruments and equipment with strong anti-interference ability and pure power besides good power supply.

3) Choose the correct implementation and cable laying: the armored shielded cable, reduce electromagnetic interference generated by the power line; the signals of different types by different cable and signal cable should be according to the transmission signal type layered laying, power supply and signal transmission at the same time is the same with different wire cable, signal cable and power cable to avoid close to parallel, to reduce electromagnetic interference.

4) Hardware filtering and software anti-interference measures: in order to reduce the common mode interference at both ends of the signal, filter is added between the two poles of the signal, and the filter can filter out most of the external interference signals, so as to effectively solve the problem of interference. At the same time, from software aspect, anti-jamming is usually done. Digital filtering and power frequency shaping sampling are usually used to eliminate periodic interference. Timing correction reference point potential can effectively prevent potential drift, and use information redundancy technology to set software protection.

5) Correct selection of grounding and perfect grounding system: the purpose of grounding is mainly to secure and suppress interference. Generally speaking, the system adopts one end grounding.

\subsection{Interference and prevention of fan monitoring system in frequency conversion harmonic environment}

1) The main factors affecting the monitoring effect under the condition of frequency conversion

Many of the current monitoring system with various temperature, power acquisition module for data acquisition, in the absence of frequency interference in the working environment, 7000 series, 9000 series, SM331 intelligent module to collect all kinds of signals are often used, stable and reliable signal acquisition system. But in the case of high power frequency conversion equipment, some signals are disturbed by the high order harmonic signals produced by the frequency conversion equipment, resulting in fluctuation and instability. Through a lot of practice analysis, in terms of the fan monitoring signal monitoring system, when the input signal into a DC voltage (+ $5 \mathrm{~V},+10 \mathrm{~V}, 0 \sim 5 \mathrm{~V})$ and current (+20mA, $4 \sim 20 \mathrm{~mA}$ ) and other types of signal acquisition, normal, almost has no frequency interference; when the input signal is RTD type (the temperature module, signal acquisition module) appeared irregular oscillations and waves, resulting in signal monitoring often appear huge fluctuation and frequent false alarm phenomenon, when the fan frequency in the environment scared when running, then returned to normal monitoring parameters. Based on the temperature acquisition module measuring circuit technology analysis, combined with the analysis of interference phenomenon of concrete application practice that such chip temperature acquisition module in the work environment, the conversion circuit of constant current power supply circuit cannot withstand high power drive and eliminate harmonic pollution generated by the motor, resulting in constant current power supply is no longer in the transverse harmonic next, resulting in 
a signal transmitting circuit becomes out of the distortion, the end result is caused by the monitoring parameters deviate from the actual value, misleading the operation status of the correct judgment of fan. Therefore, from this point of view, the key to solve the problem of distortion of temperature parameters in frequency conversion environment is to solve the interference to the constant current power of the acquisition module, and ensure that the transverse flow power is kept constant.

2) Several methods to deal with the distortion of the disturbed temperature in the fan monitoring system

Through long-term research and practice, under the premise of conventional anti-interference measures, several effective methods have been formed for fan monitoring system to deal with temperature distortion. Now these methods are briefly introduced and compared.

3) Temperature distortion processing by TT4C temperature acquisition module

Generally speaking, the interference signals generated by the frequency conversion harmonics have little interference with the DC current and the voltage signal, but the interference of the resistance signals is larger. Therefore, the temperature signal can be collected and processed jointly by the TT4C temperature acquisition module and the ordinary analog acquisition module, and the hardware wiring diagram, as shown in 3.5. TT4C temperature transmission module is the use of this design principle, 4 channel as Beijing Kunlun coast sensor research center developed 3 wire temperature transmit module, TT4C module for measuring range -50 to 200 DEG C, the PT100 resistance temperature sensor signal conversion is $0 \sim 5 \mathrm{~V}$ DC voltage and output by simulation data acquisition module collects, the communication mode is uploaded to the monitoring computer. Through the linear transformation of temperature and voltage, the actual temperature value is obtained, which can solve the interference problem of the frequency conversion harmonic signal to the temperature signal of the monitoring system. The field application shows that the method is feasible.

But this mode also has shortcomings: when the frequency converter runs below $35 \mathrm{~Hz}$, the effect of harmonic interference is not good, so this mode is generally suitable for $35 \sim 50 \mathrm{~Hz}$ environment, and its application conditions are limited.

4) Adding temperature transmitter to eliminate temperature distortion

A popular treatment temperature distortion measure is used with variable temperature transmitter to send function, TH series temperature control instrument Japan Toupie electric transmission, display and alarm function, strong anti-interference ability, suitable for all kinds of common thermocouples and thermal resistance temperature sensor. Therefore, the $\mathrm{TH}$ temperature transmitting instrument acquisition and conversion of temperature signal, the standard signal through the conversion circuit of the acquisition, so it will be directly connected to the PT100 resistance temperature sensor signal to the transmitter terminal, converted to $4 \sim 20 \mathrm{~mA}$ current signal, through the electricity collecting module will be $4 \sim 20 \mathrm{~mA}$ into electrical signals computer

Received digital signal to achieve temperature acquisition and processing.

The industrial practice shows that the accuracy of the temperature data collected by the TH series temperature transmitter is high. Compared with the measured data, the error can be controlled within $0.5 \%$. But in places where there is no need for digital display, if we adopt this way, we need to increase the digital display meter, so that the system cost will increase correspondingly. At the same time, the cost of TH temperature transmitter is higher. To achieve this kind of anti-interference processing, we will bring a lot of waste of money.

5) Temperature measurement module and capacitive filter facility to eliminate temperature distortion

Low frequency interference is a problem that can't be solved by the combination of TT4C and power acquisition module. The main reason is that the harmonic pollution of frequency conversion system itself has not been well handled and suppressed. Therefore, starting from the idea of restraining the source of harmonic interference, a temperature acquisition module is generated directly through the common temperature acquisition module, and a certain capacitance filter is processed in the process of signal acquisition. The operation mode is to connect large one and two small filter capacitors in the temperature signal acquisition loop. The large capacity capacitor is 
used to eliminate the low frequency harmonics, and the small capacitance is used to eliminate the high-frequency harmonics.

This method effectively solves the problem that the ordinary temperature acquisition module cannot collect the true temperature under the low frequency condition, and the operation mode is simple and the implementation cost is low.

\subsection{Design of anti-interference measures for monitoring system}

Due to the construction of the ventilation system and the need to maintain many different units jointly carry out installation, anti-jamming design, maintenance and other work by the inverter supply unit is responsible for the inverter, in view of the important functions of the monitoring system of ventilator in mine ventilation system, the independence and stability of the monitoring system must ensure that the ventilation, to prevent because of external the interference caused by affecting production. Therefore, the anti-jamming design scheme takes the monitoring system as the core, which is based on the whole protection, and focuses on the filtering process of the interference parts. On the basis of the above principles, the comparison and analysis of several methods to solve the temperature distortion are made, and the anti-interference measures of the fan on-line monitoring system are designed.

1) Select common temperature module and capacitance filter to eliminate temperature distortion. The system selects 8 modules to complete the collection and anti-interference protection of the 20 temperature signal. The filter circuit is welded on the integrated circuit board, and the special output terminal is made, which is convenient for installation and maintenance.

2) Input module and output module's signal line all use shielded line, shielding layer is good grounded. Generally, one end grounding is used to avoid the loop current caused by potential difference in the location, resulting in interference.

3) Signal line, power line, power line, inverter main circuit control line adopts layered arrangement, each with a trough which separated from the inverter main circuit control line and control line should be kept at a distance of more than $20 \mathrm{~cm}$, the interference is more serious situations, should control loop cable pipe, to cut off the radiation interference.

4) The signal line grounding and the system protection grounding are separated. The ground wires of all walks of life ensure good grounding. The ground wire is thick and short. Generally, the copper wire with a section area larger than $10 \mathrm{~mm} 2$ is firmly connected to the ground terminal.

5) A short connection of resistance is adopted for the unused input channel in the signal input module to avoid crosstalk between channels.

6) The signal acquisition module uses DC power supply (the anti-interference ability of AC power supply is weak), and the output of the acquisition module uses DC voltage or current.

7) If there are non-isolated sensors on the scene, in order to prevent the potential difference between the input channel measurement line $\mathrm{M}$ - and the reference point of the measuring circuit (the common mode potential) exceeds the allowable maximum value, the equal potential is used to connect the conductor between the measuring point and the reference point.

\section{References}

[1] Yang J. Application of PLC Variable Frequency Speed Control System in Coal Mine Ventilator [J]. Shanxi Coking Coal Science \& Technology, 2015, 114(36):9886-92.

[2] Bristle T J, Collins S, Hewer I, et al. Anesthesia and critical care ventilator modes: past, present, and future. [J]. Aana Journal, 2014, 82(5):387-400.

[3] Yin J L, Liu D C, Liao S B, et al. Application of Frequency Conversion Speed Regulating Technology in Reducing the Ventilator Energy Consumption [J]. Mechanical \& Electrical Engineering Technology, 2013.

[4] Sud S, Sud M, Friedrich J O, et al. High frequency oscillation in patients with acute lung injury and acute respiratory distress syndrome (ARDS): systematic review and meta-analysis.[J]. Bmj, 
2010, 340(7759):1290-1290.

[5] Li N. Application of Frequency Conversion Speed Adjustment Based on PLC Control in Mine Ventilation Machine [J]. Journal of Henan Science \& Technology, 2015.

[6] Yang J. Application of PLC Variable Frequency Speed Control System in Coal Mine Ventilator [J]. Shanxi Coking Coal Science \& Technology, 2015, 114(36):9886-92.

[7] Piper A. Obesity hypoventilation syndrome: therapeutic implications for treatment. [J]. Expert Review of Respiratory Medicine, 2010, 4(1):57-70.

[8] Branson R D. Faulty ventilator and functionally crossed pipelines [J]. Anaesthesia, 2010, 48(3):270-270.

[9] Biro P, Spahn D R, Pfammatter T. High-frequency jet ventilation for minimizing breathing-related liver motion during percutaneous radiofrequency ablation of multiple hepatic tumours [J]. British Journal of Anaesthesia, 2009, 102(5):650-3. 